\title{
Nonequilibrium Electron and Lattice Dynamics of Strongly Correlated Quantum Materials
}

\author{
T. Konstantinova ${ }^{1}$, Lijun $\mathrm{Wu}^{1}$, Junjie $\mathrm{Li}^{1}$, Jing Tao ${ }^{1}$, G. Gu ${ }^{1}$, C. Petrovic ${ }^{1}$, Xiaozhe Shen ${ }^{2}$, Xijie Wang ${ }^{2}$ \\ and Yimei Zhu ${ }^{1}$
}

${ }^{1}$ Brookhaven National Laboratory, Upton, New York, United States, ${ }^{2}$ SLAC National Accelerator Laboratory, Menlo Park, California, United States

The interplay between the electronic and lattice degrees of freedom in non-equilibrium states of strongly correlated systems has been attracting controversy for decades. While progress has been made in establishing a hierarchy of electronic interactions with the use of time-resolved techniques, the role of the phonons often remains in dispute, a situation highlighting the need for tools that directly probe the lattice. Here we present the first combined $\mathrm{MeV}$ ultrafast electron diffraction (MeV-UED) and time- and angleresolved photoemission spectroscopy (tr-APRES) study of optimally doped $\mathrm{Bi}_{2} \mathrm{Sr}_{2} \mathrm{CaCu}_{2} \mathrm{O}_{8}+\delta$. Quantitative analysis of the intensity changes of Bragg and superlattice reflections as well as thermal diffuse scattering (Fig.1) at various time delays after photoexcitation reveals how the energy is transferred from electrons to phonons and from one phonon branch to others along with the time scales related to the path of energy thermalization within the lattice. Bloch wave simulations show that the energy absorbed by electrons is transferred to $\mathrm{Cu}-\mathrm{O}$ breathing phonons only (Fig.1e). Thus, the electron-phonon coupling on the sub-picosecond time scale is anisotropic and is limited to the ab-plane. The lowest energy acoustic branches with correlated atomic motions along the $<110>$ directions form on a 10ps scale, a characteristic time much slower than the dynamics of the electronic states. Detailed analysis of the lattice and electron subsystems' dynamics provides a unified picture of nonequilibrium electron-phonon interactions in the cuprates beyond the N-temperature model.

Another system we report is FeSe, the simplest iron-chalcogenide superconductor. At low temperature it undergoes a transition to the nematic electronic state, deemed a precursor of superconductivity, which is accompanied by a weak change in the average crystal symmetry from tetragonal P4/nmm to orthorhombic Cmma group. To understand the formation of electronic nematicity, which is a common thread of unconventional superconductors, we use MeV-UED to systematically probe the lattice interactions with electronic degrees of freedom in its superconducting state and find a significant lattice response to local nematicity (Fig.2). We observe that a perturbation by a laser pulse leads to a surprising enhancement of the high-symmetry crystalline order as a result of suppression of low-symmetry local lattice distortions, which are signatures of nematic fluctuations. The distortions are present at temperatures both below and above the nematic phase transition, as corroborated by our synchrotron x-ray pair distribution function (PDF) analysis and transmission electron microscopy measurements. Nonequilibrium lattice behavior of FeSe reveals two distinct time scales of nematic response to photoexcitation, 130 ps and 40 ps, corresponding to diffusive and percolative dynamics of nematic fluctuations, respectively. The observed structural response, which is naturally explained by redistribution of the relative population of the two low- and high-symmetry phases (Fig.2e), sheds new light on the formation of the nematic phase from imperfect ordering of its fluctuations in FeSe and other layered systems and stimulates further theoretical development towards full understanding of nematicity [3]. 


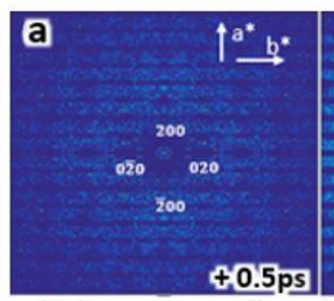

High-energy mode

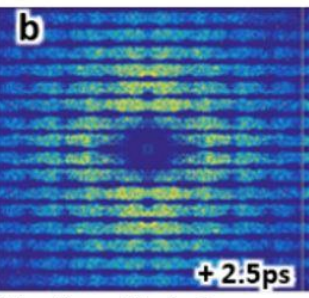

Mostly optical phonons

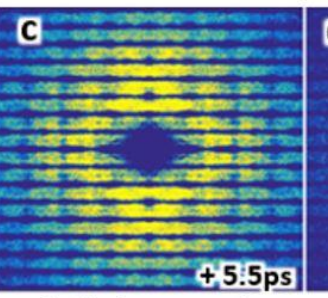

Optical + acoustic

\section{d}

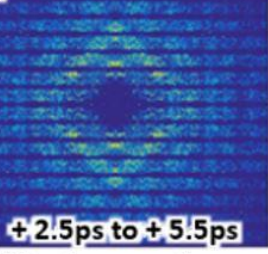

Mostly acoustic

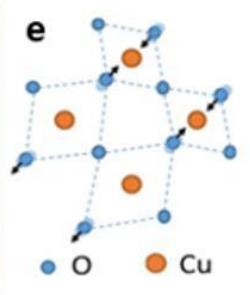

- $\mathrm{O} \mathrm{Cu}$

Figure 1. Analysis of thermal diffuse scattering (TDS) dynamics at $30 \mathrm{~K}$ using UED in $\mathrm{Bi}_{2} \mathrm{Sr}_{2} \mathrm{CaCu}_{2} \mathrm{O}_{8}+\delta$ superconductor. (a-d) TDS images obtained by subtracting intensities before photoexcitation at certain delays after the laser pulse arrival. (a) high-energy mode, (b) mostly optical phonons, (c) optical and acoustic phonons, and (d) mostly acoustic phonons with correlated atomic motions along <110> directions. Dark horizontal lines are depleted intensity of Bragg and superlattice peaks after their removal. (e) Schematic motion of atoms for the half-breathing phonon mode.
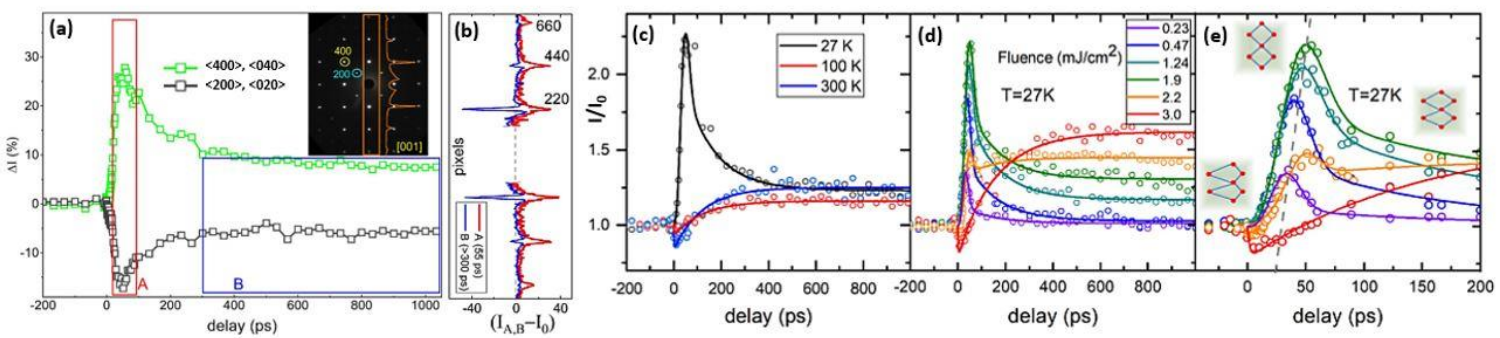

Figure 2. (a) Bragg peak dynamics for (200) and (400) reflections of FeSe superconductor. Inset shows the diffraction pattern. The orange line in the inset shows the diffraction intensity profile integrated within the indicated frame. (b) Averaged intensity differences from frames A (red) and B (blue) in (a) after the pump pulse with respect to the profile of the unpumped sample. (c)Dynamics of (800) peaks obtained at different temperatures for the incident fluence of $1.65 \mathrm{~mJ} / \mathrm{cm} 2$. (d) Dynamics of the (800) peaks at different excitation fluences for the full range of $1000 \mathrm{ps}$ and (e) during the first $200 \mathrm{ps}$ at $27 \mathrm{~K}$. The gray dashed line in (e) is a guide to eye. Insets show schematics of unequal atomic bonds at the corresponding time intervals. Open circles are the experimental data and solid lines are the fits.

\section{References}

[1] Konstantinova, T., et al., "Non-equilibrium electron and lattice dynamics of strongly correlated $\mathrm{Bi}_{2} \mathrm{Sr}_{2} \mathrm{CaCu}_{2} \mathrm{O}_{8}+\delta$ single crystals", Sci. Adv., 4: eaap7427 (2018).

[2] Konstantinova, T., et al., "Photoinduced dynamics of nematic order parameter in FeSe", Phys. Rev. B 99, 180102(R) (2019) (Rapid Communication; Editors' Suggestion).

[3] Work at BNL was supported by the US DOE-BES, MSED, under Contract No. DESC0012704, and at SLAC was supported by DOE-BES User Facility under Contracts No. DE-AC02-05-CH11231 and No. DE-AC02-76SF00515. 